\title{
Genetic transcriptional regulatory model driven by the time-correlated noises
}

\author{
Xiao-miao Zhang, Bao-quan Ai,* and Jian-wen Xiong \\ Laboratory of Quantum Information Technology, ICMP and SPTE, \\ South China Normal University, 510631 Guangzhou, China
}

(Received on 29 October, 2009)

\begin{abstract}
Steady state properties of a kinetic model of Smolen-Baxter-Byrne [P. Smolen, D. A. Baxter, J. H. Byrne, Amer. J. Physiol. Cell. Physiol. 274, 531 (1998)] are investigated in presence of two time-correlated noises. The steady state probability distribution can be obtained by solving the Fokker-Planck equation. It is found that both the correlated-time between the white noise and the colored noise and that between the colored noises can convert the bistability to monostability while the former activates the transcription and the latter suppresses it.
\end{abstract}

Keywords: genetic transcriptional regulatory mechanism, steady-state probability distribution, time-correlated noises, FokkerPlanck equation.

\section{INTRODUCTION}

Recently, nonlinear stochastic systems with noise term have been the subject of extensive investigations. Noise can induce many significant phenomena such as phase transition and stochastic resonance. The concept of noise-induced transition has many applications in the fields of physics, chemistry and biology [1-27]. Especially in biologic system, it is very important to explore the effects caused by noise in nonlinear system and to find the condition, causation and application, which can give a strong impulse on the development and the intercross of subjects.

Gene expression and regulation processes is the core problem of molecular biology and a hot subject in biology study. Genetic transcriptional regulatory mechanism is a simulation of the biologic gene regulatory system in which the transcription factor accelerates the transcription process. There are some studies about this simulation [28, 39, 40]. Nevertheless, in the previous work, they took the correlation-time as zero. Actually, the real correlation-time is very small, but it is not equal to zero exactly. So taking finite correlation-time into consideration may be more reasonable. Generally, analyzing the evolvement of a system with nonzero correlationtime may reflect the dynamic effects of a noise on a system more completely. So it may make some exploring sense to study the effects of the correlation-time on the gene transcription regulatory mechanism.

In this paper, based on the kinetic model of SmolenBaxter-Byrne [29], the effects of two time-correlated noises (the fluctuations in the transcription rate and basal rate of synthesis of transcription factor) have been investigated through the numerical computation and the analysis theory. It is found that the additive colored noise reduces the transcription efficiency. Remarkably, the correlated-time between the additive colored noises as well as the multiplicative white noise can convert the bistability to monostability and can suppress the transcription while the correlation-time between the multiplicative white noise and the additive colored noise can convert the bistability to monostability and can activate the transcription, which has not been found before.

\section{THE MODEL OF GENE TRANSCRIPTION}

Now there are mainly two kinds of theoretical models of gene transcriptional regulatory mechanism: positive transcription control and negative transcription control. Smolen et al. have proposed a model simulation about the transcription factor facilitating the gene transcription in 1998 [29], which contains dimer, positive feedback of transcription factor and nonlinear reciprocity. The model considers a single transcriptional activator (TF-A) as part of a pathway mediating a cellular response to a stimulus. The TF forms a homodimer that can bind to responsive elements (TF-REs). The tf-a gene incorporates a TF-RE, and when homodimers bind to this element TF-A transcription is increased. Binding to the TF-REs is independent of dimer phosphorylation. However, only phosphorylated dimers can activate transcription. The fraction of phosphorylated dimers depends on the activity of kinases and phosphatases whose activity can be regulated by external signals. Therefore, this model incorporates both signal-activated transcription and positive feedback on the rate of TF synthesis. In order to capture the salient features of TF dimerization, binding, and phosphorylation-dependent regulation of transcription and to make the model appreciated intuitively, some simplifications were made [30]. It is assumed that the transcription rate saturates with TF-A dimer concentration to maximal rate $\alpha$, which is proportional to TF-A phospholation. The synthesis rate is $\beta$ at negligible dimer concentration. TF-A is eliminated with a rate constant $\kappa_{2}$. The basic mechanism is shown in Fig. 1 [29].

According to several biologic and chemical reactions of this model, a differential equation about the evolvement of concentration of transcription factor with time can be acquired [28]:

$$
\dot{x}=\frac{\alpha x^{2}}{x^{2}+\kappa_{1}}-\kappa_{2} x+\beta,
$$

where $x$ is the concentration of transcription factor (TF-A), $\kappa_{1}$ is the concentration of dimer which is not adhering to the responsive elements (termed as TF-REs). For certain values of $\alpha(\alpha<5.45$ or $\alpha>6.68)$ there is one unique steady solution of [TF-A] (the concentration of transcription factor) [28]. For other values of $\alpha$, there are three steady solutions with one unstable state and the other two stable states.

But this model is a deterministic model. Many experiments indicated that gene transcription is a stochastic process. So it is necessary to revise it. Liu and co-workers 


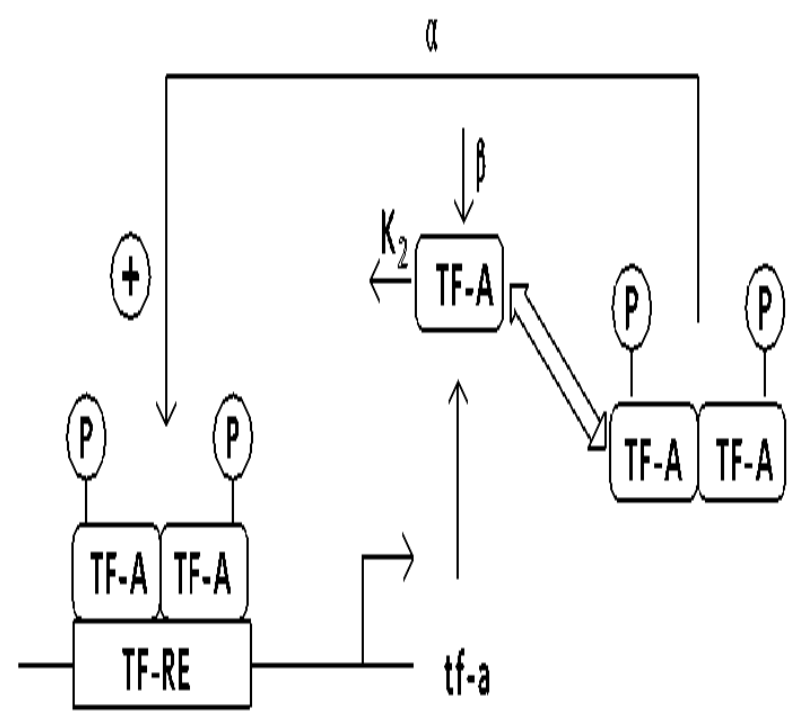

FIG. 1: The schematic description of positive autoregulatory model [29]. TF-A denotes transcription factor, TF-RE responsive element, $\alpha$ transcription rate, $\beta$ the basal rate of synthesis, $P$ phosphorylated, $\kappa_{2}$ the degradation rate.

have studied the effects of two Gaussian white noises on the steady state distribution of protein concentration [28]. But they did not consider the correlation time. In this paper, we just study it. In order to introduce these effects, we rewrite the Eq. (1) as follows,

$$
\dot{x}=\frac{\alpha x^{2}}{x^{2}+\kappa_{1}}-\kappa_{2} x+\beta+\frac{x^{2}}{x^{2}+\kappa_{1}} \xi(t)+\eta(t),
$$

where $\xi(t)$ is a white noise and $\eta(t)$ is a colored noise with the following properties [31]:

$$
\begin{gathered}
\langle\eta(t)\rangle=\langle\xi(t)\rangle=0, \\
\left\langle\xi(t) \xi\left(t^{\prime}\right)\right\rangle=2 D_{1} \delta\left(t-t^{\prime}\right), \\
\left\langle\eta(t) \eta\left(t^{\prime}\right)\right\rangle=\frac{D_{2}}{\tau_{1}} \exp \left[-\frac{\left|t-t^{\prime}\right|}{\tau_{1}}\right], \\
\left\langle\eta(t) \xi\left(t^{\prime}\right)\right\rangle=\left\langle\xi(t) \eta\left(t^{\prime}\right)\right\rangle=\frac{\lambda \sqrt{D_{1} D_{2}}}{\tau_{2}} \exp \left[-\frac{\left|t-t^{\prime}\right|}{\tau_{2}}\right],
\end{gathered}
$$

here $D_{1}$ is the intensity of white noise, $\tau_{1}$ and $D_{2}$ are the correlation time and intensity of the multiplicative colored noise, and $\tau_{2}$ is the noise correlation time of the coupling betweem the multiplicative and additive noise. In the limit $\tau_{1} \rightarrow 0$, the additive noise tends to white noise, while the coupling between the multiplicative and additive noise is colored correlated.

In this paper, we emphasize on finding how the correlation time affects the genetic transcriptional regulatory model. In the following part we give a steady state analysis of the model.

\section{STEADY STATE ANALYSIS OF THE MODEL}

If a nonlinear system contains a colored noise, it is a nonMarkov stochastic process. It is necessary to develop some approximate methods to transform the non-Markov process to the Markov process in order to get analytic results. The functional analysis and the unified colored noise approximation (UCNA) are the methods commonly used in the analysis [19, 32-37]. According to the stochastic Liouville equation, Eq. (2) satisfies

$$
\begin{aligned}
& \frac{\partial P(x, t)}{\partial t}=-\frac{\partial}{\partial x} h(x) P(x, t) \\
& -\frac{\partial}{\partial x} g_{1}(x)\langle\xi(x) \delta(x(t)-x)\rangle-\frac{\partial}{\partial x} g_{2}(x)\langle\eta(t) \delta(x(t)-x)\rangle,
\end{aligned}
$$

where

$$
\begin{gathered}
h(x)=\frac{\alpha x^{2}}{x^{2}+\kappa_{1}}-\kappa_{2} x+\beta, \\
g_{1}(x)=\frac{x^{2}}{x^{2}+\kappa_{1}}, \\
g_{2}(x)=1,
\end{gathered}
$$

here the probability distribution function can be expressed as $P(x, t)=\langle\delta(x(t)-x)\rangle$.

By applying the Novikov theorem [38] and Fox's approach $[19,32,34,35]$, the approximate Fokker-Planck equation corresponding to Eq. (7) can be written as

$$
\begin{aligned}
& \frac{\partial P(x, t)}{\partial t}=-\frac{\partial}{\partial x} h(x) P(x, t)+ \\
& D_{1} \frac{\partial}{\partial x} g_{1}(x) \frac{\partial}{\partial x} g_{1}(x) P(x, t)+D_{2} \frac{\partial}{\partial x} g_{2}(x) \frac{\partial}{\partial x} g_{2}(x) P(x, t)+ \\
& \frac{\lambda \sqrt{D_{1} D_{2}}}{1-\tau_{2}\left[h^{\prime}\left(x_{s}\right)-\frac{g_{2}^{\prime}\left(x_{s}\right)}{g_{2}\left(x_{s}\right)} h\left(x_{s}\right)\right]} \frac{\partial}{\partial x} g_{1}(x) \frac{\partial}{\partial x} g_{2}(x) P(x, t)+ \\
& \frac{\lambda \sqrt{D_{1} D_{2}}}{1-\tau_{2}\left[h^{\prime}\left(x_{s}\right)-\frac{g_{1}^{\prime}\left(x_{s}\right)}{g_{1}\left(x_{s}\right)} h\left(x_{s}\right)\right]} \frac{\partial}{\partial x} g_{2}(x) \frac{\partial}{\partial x} g_{1}(x) P(x, t) .(11)
\end{aligned}
$$

Where 


$$
x_{s}=\sqrt[3]{-\frac{q}{2}+\sqrt{\left(\frac{q}{2}\right)^{2}+\left(\frac{p}{3}\right)^{3}}}+\sqrt[3]{-\frac{q}{2}-\sqrt{\left(\frac{q}{2}\right)^{2}+\left(\frac{p}{3}\right)^{3}}}-\frac{b}{3}
$$

$$
\begin{gathered}
p=\kappa_{1}-\frac{(\alpha+\beta)^{2}}{3 \kappa_{2}^{2}}, \\
q=\frac{1}{27}\left(\frac{\alpha+\beta}{\kappa_{2}}\right)^{3}+\frac{p}{3} \frac{\alpha+\beta}{\kappa_{2}}-\frac{\kappa_{1}}{\kappa_{2}} \beta, \\
b=-\frac{\alpha+\beta}{\kappa_{2}} .
\end{gathered}
$$

From Eq. (11), one has

$\frac{\partial P(x, t)}{\partial t}=-\frac{\partial}{\partial x}\left[h(x)+g^{\prime}(x) g(x)\right] P(x, t)+\frac{\partial^{2}}{\partial x^{2}} g(x) g(x) P(x, t)$,

where
If the multiplicative noise is colored with finite correlation time $\tau_{1}$, the UCNA method can be employed. Thus the non-Markov process of Eq. (2) can be simplified to a onedimensional Markov process

$$
\frac{d x}{d t}=\frac{1}{A\left(x, \tau_{1}\right)}\left[h(x)+g_{2}(x) \Gamma(t)+g_{1}(x) \xi(t)\right]
$$

with

$$
\begin{aligned}
A\left(x, \tau_{1}\right) & =1-\tau_{1}\left[h^{\prime}(x)-\frac{g_{2}^{\prime}(x)}{g_{2}(x)} h(x)\right] \\
& =1-\tau_{1}\left[\frac{2 \alpha \kappa_{1} x}{\left(x^{2}+\kappa_{1}\right)^{2}}-\kappa_{2}\right],
\end{aligned}
$$

where $h^{\prime}(x)$ and $g_{2}^{\prime}(x)$ are the derivatives of $h(x)$ and $g_{2}(x)$ with respect to $x$ and $\Gamma(t)$ is white noise with

$$
\langle\Gamma(t)\rangle=0,\left\langle\Gamma(t) \Gamma\left(t^{\prime}\right)\right\rangle=2 D_{2} \delta\left(t-t^{\prime}\right) .
$$

By combining the results, Eq. (11), of the functional analysis and Eq. (18) of the UCNA, the Fokker-Planck equation corresponding to Eqs. $(2,3,4,5,6)$ can be written as

$$
\begin{aligned}
\frac{\partial P(x, t)}{\partial t}= & -\frac{\partial}{\partial x} \frac{h(x)}{A\left(x, \tau_{1}\right)} P(x, t)+D_{2} \frac{\partial}{\partial x} \frac{g_{2}(x)}{A\left(x, \tau_{1}\right)} \frac{\partial}{\partial x} \frac{g_{2}(x)}{A\left(x, \tau_{1}\right)} P(x, t) \\
& +\frac{\lambda \sqrt{D_{1} D_{2}}}{1-\frac{\tau_{2}}{A\left(x_{s}, \tau_{1}\right)}\left[h^{\prime}\left(x_{s}\right)-\frac{g_{1}^{\prime}\left(x_{s}\right)}{g_{1}\left(x_{s}\right)} h\left(x_{s}\right)\right]} \frac{\partial}{\partial x} \frac{g_{2}(x)}{A\left(x, \tau_{1}\right)} \frac{\partial}{\partial x} \frac{g_{1}(x)}{A\left(x, \tau_{1}\right)} P(x, t) \\
& +D_{1} \frac{\partial}{\partial x} \frac{g_{1}(x)}{A\left(x, \tau_{1}\right)} \frac{\partial}{\partial x} \frac{g_{1}(x)}{A\left(x, \tau_{1}\right)} P(x, t) \\
& +\frac{\lambda \sqrt{D_{1} D_{2}}}{1-\frac{\tau_{2}}{A\left(x_{s}, \tau_{1}\right)}\left[h^{\prime}\left(x_{s}\right)-\frac{g_{2}^{\prime}\left(x_{s}\right)}{g_{2}\left(x_{s}\right)} h\left(x_{s}\right)\right]} \frac{\partial}{\partial x} \frac{g_{1}(x)}{A\left(x, \tau_{1}\right)} \frac{\partial}{\partial x} \frac{g_{2}(x)}{A\left(x, \tau_{1}\right)} P(x, t) .
\end{aligned}
$$

Thus the two different kinds of colored noise can be approximated and expressed by Eq. (21). It should be mentioned that the regime of the approximation method used in deriving
Eq. (21) is [32]

$$
G\left(x, \tau_{1}, \tau_{2}\right)=1-\frac{\tau_{2}}{A\left(x, \tau_{1}\right)}\left[h^{\prime}\left(x_{s}\right)-\frac{g_{2}^{\prime}\left(x_{s}\right)}{g_{2}\left(x_{s}\right)} h^{\prime}\left(x_{s}\right)\right]>0,
$$




$$
G\left(x, \tau_{1}, \tau_{2}\right) \gg \lambda \sqrt{D_{1} D_{2}}\left|\frac{h^{\prime}(x)}{h(x)}\right| .
$$

In the following calculations, these conditions are satisfied. Thus the corresponding Fokker-Planck equation of the steady state probability distribution system, Eq. (21) when $\partial P(x, t) / \partial t=0$, can be written as

$$
P_{s t}(x)=\exp \left[\int \frac{H(x)}{G^{2}(x)} d x-\ln |G(x)|\right],
$$

with

$$
H(x)=\frac{h(x)}{A\left(x, \tau_{1}\right)}, G(x)=\frac{g(x)}{A\left(x, \tau_{1}\right)} .
$$

Then we give a numerical analysis for equation (24) and the results are presented in Figs. 2-5.



FIG. 2: $P_{s t}$ (steady probability density) as a function of $x$ for different values of the multiplicative white noise intensity $D_{1} . D_{1}=0.1$, $0.3,0.5$, and 0.7 , respectively, at $D_{2}=0.5, \tau_{1}=0.1, \tau_{2}=0.5, \lambda=0.4$, $\alpha=5.75, \beta=0.4, \kappa_{1}=10.0$, and $\kappa_{2}=1.0$. (units are relative).

The effects of the strength of the multiplicative white noise $D_{1}$ (the fluctuation of transcription rate) on the steady state probability distribution (SPDF) are plotted in Fig. 2. As we can see from the curves, for a small value of $D_{1}$, the system has two steady states with the upper state excelling the lower state. With the increasing of the noise strength, the height of the right peak decreases while the height of the left peak increases. For a large noise strength the height of the right peak decreases drastically. Therefore, the multiplicative white noise can drive the steady state from the upper state to the lower state, and the system from bistability to monostability. That is to say, strong fluctuation of transcription rate may suppress the gene transcription.

Fig. 3 depicts the effects of the strength of the additive colored noise $D_{2}$ (the fluctuation of basal rate of synthesis) on the steady state probability distribution (SPDF). For a small value of $D_{2}$, the system has two steady states with the lower



FIG. 3: $P_{s t}$ (steady probability density) as a function of $x$ for different values of the additive colored noise intensity $D_{2} . D_{2}=0.1$, $0.3,0.5$, and 0.7 , respectively, at $D_{1}=0.1, \tau_{1}=0.1, \tau_{2}=0.5, \lambda=0.4$, $\alpha=5.75, \beta=0.4, \kappa_{1}=10.0$, and $\kappa_{2}=1.0$. (units are relative).

state excelling the upper state. With the increasing of the noise strength, the height of the left peak and the right peak decrease simultaneously. The peak becomes flat for big noise strength. So it can be prophesied that the system turns into confusion for large perturbation of environment. This implies that the additive colored noise do not affect the transcription switch, but make a strong impact on the transcription efficiency. That is to say, the fluctuation of basal rate of synthesis can be used to weaken the transcription efficiency. Such a development could have important implications for gene transcription regulation.

Fig. 4(a)-4(c) are plots of the effects of different values of $\tau_{1}$ (the correlation time of the colored noise) on the steady state probability distribution. For a small value of $\tau_{1}$, the curves have a similar feature as Fig. 2 when $D_{1}$ is small. With the increasing of the value of a small value of $\tau_{1}$ (see in Figs. 4a, 4b), the height of the left peak and the height of the right peak increase simultaneously and the height of the right peak increases more slowly than the left one. For a large value of $\tau_{1}$ (see in Fig. 4c), with the increasing of the value of the $\tau_{1}$, the height of the right peak decreases while the height of the left one increases extremely rapidly. Therefore, the correlated time of the colored noise can drive the steady state from the upper state to the lower state, and the system from bistability to monostability. That is to say, strong fluctuation of correlation time of the multiplicative colored noise (the transcription rate) may suppress the gene transcription.

In Fig. 5, we show the effects of different values of $\tau_{2}$ (the correlation time between the additive colored noise and the multiplicative white noise) on the steady state probability distribution. When $\tau_{2}=0$, the system exhibits a typical bistable region. There are two peaks in the distribution curve: the right one has a lower concentration site and the left one has a higher concentration site. Hence, the system 

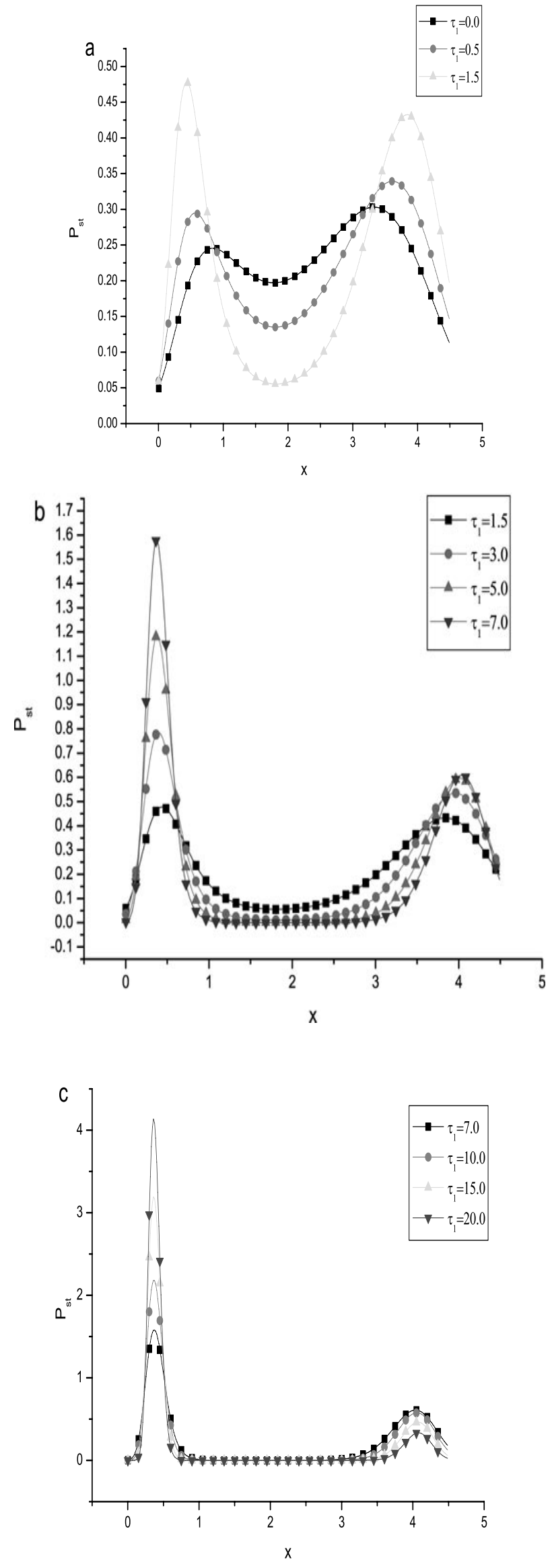

FIG. 4: $P_{s t}$ (steady probability density) as a function of $x$ for different values of the correlation time of the multiplicative color noise $\tau_{1}$. Fig. 4(a): $\tau_{1}=0.0,0.5$, and 1.5; Fig. 4(b): $\tau_{1}=1.5,3.0,5.0$, and 7.0; Fig. 4(c): $\tau_{1}=7.0,10.0,15.0$, and 20.0, respectively, at $D_{1}=0.1$, $D_{2}=0.1, \tau_{2}=0.5, \lambda=0.5, \alpha=5.5, \beta=0.5, \kappa_{1}=10.0$, and $\kappa_{2}=1.0$. (units are relative).

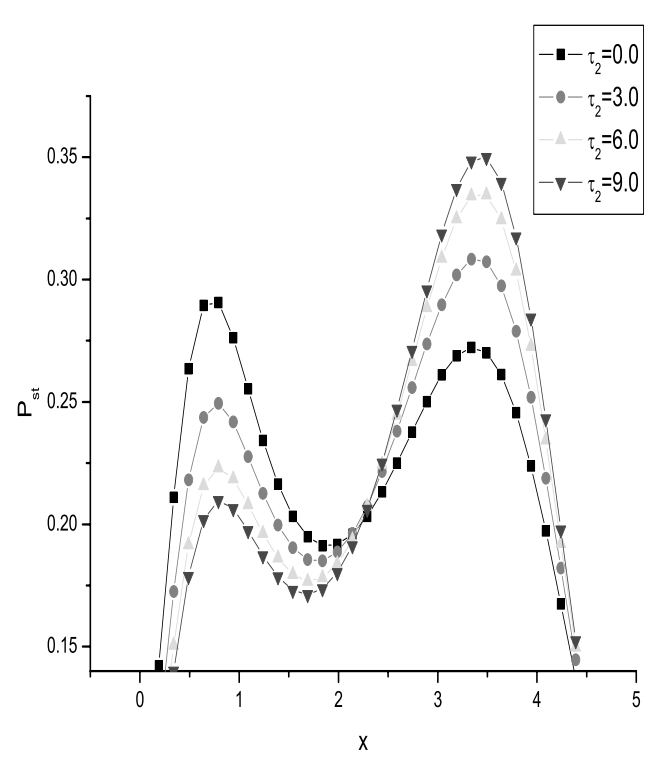

FIG. 5: $P_{s t}$ (steady probability density) as a function of $x$ for different values of the correlation time between the multiplicative noise and the additive noise $\tau_{2} . \tau_{2}=0.0,3.0,6.0$ and 9.0, respectively, at $D_{1}=0.1, D_{2}=0.1, \tau_{1}=0.1, \lambda=0.5, \alpha=5.5, \beta=0.5, \kappa_{1}=10.0$, and $\kappa_{2}=1.0$. (units are relative).

has two steady states. With the increasing of the value of $\tau_{2}$, the height of the right peak increases while the height of the left one reduces. Therefore, the correlation time between the additive colored noise and the multiplicative white noise can drive the steady state from the lower state to the higher state, and the system from bistability to monostability. That is to say, strong fluctuation of correlation time between the additive colored noise (basal rate of synthesis) and the multiplicative white noise (the transcription rate) can activate the gene transcription.

\section{CONCLUSIONS AND SUMMARY}

In this paper, based on the kinetic model of SmolenBaxter-Byrne [29], the effects of the fluctuations in the transcription rate and basal rate of synthesis of transcription factor have been investigated through the numerical computation and the analysis theory. It is found that the additive colored noise reduces the transcription efficiency. Remarkably, the correlated-time between the additive colored noises as well as the multiplicative white noise can convert the bistability to monostability and can suppress the transcription. The correlation-time between the multiplicative white noise and the additive colored noise can convert the bistability to monostability and can activate the transcription.

The work was supported by the National Natural Science Foundation of China under Grant No. 30600122 and GuangDong Provincial Natural Science Foundation under Grant No. 06025073. 
[1] A. Fulinski and T. Telejko, Phys. Lett. A 152, 11 (1991).

[2] B. Q. Ai, X. J. Wang, G. T. Liu, L. G. Liu, Phys. Rev. E 67, 022903 (2003).

[3] B. Q. Ai and L. G. Liu, J. Phys. Chem. B 112, 9540 (2008).

[4] J. M. Sancho, M. San Miguel, S. L. Katz, and J. D. Gunton. Phys. Rev. A 26, 1958 (1982).

[5] B. Q. Ai and L. G. Liu, Phys. Rev. E 74, 051114 (2006).

[6] R. Benzi, A. Sutera, and A. Vulpiani, J. Phys. A 14, L453 (1981).

[7] R. Benzi, G. Parisi, A. sutera, and A. Vulpiani, Tellus 34, 10 (1982).

[8] B. McNamara and K. Wiesenfeld, Phys. Rev. A 39, 4854 (1989).

[9] T. Zhou and F. Moss, Phys. Rev. A 41, 4255 (1990).

[10] P. Jung and P. Hanggi., Phys. Rev. A 44, 8032 (1991).

[11] G. Hu. T. Ditzinger, C. Z. Ning, and H. Haken, Phys. Rev. Lett. 71,807 (1993).

[12] M. I. Dykman, R. Mannella, P. V. E. McClintock, and N. G. Stocks, Phys. Rev. lett. 68, 2985 (1992).

[13] A. Neiman and L. Schimansky-Geier, Phys. Rev. Lett. 72, 2988 (1994).

[14] P. Jung and G. Maye-Kress, Phys. Rev. Lett. 74,2130 (1995).

[15] L. Ganna, F. Marchesoni, and S. Santucci, Phys. Rev. Lett. 74, 1052 (1995)

[16] J. F. Lindner, B. K. Meadows, W. L. Ditto, M. E. Inchiosa, and A. R. Bulsara, Phys. Rev. Lett. 75, 3 (1995).

[17] G. Hu, H. Haken, and F. Xie, Phys. Rev. Lett. 77, 1925 (1996).

[18] A. R. Bulsara and L. Gammaitoni, Phys. Today 49 (3), 39 (1996).

[19] L. Gammaitoni, P. Hanggi, P. Jung, and F. Marchesoni, Rev. Mod. Phys. 70, 224 (1998).

[20] V. Berdichevsky and M. Gitterman, Phys. Rev. E 60, 1494
(1999).

[21] F. Marchesoni, F. Apostolico, and S. Santucci, Phys. Rev. E 59, 3958 (1999).

[22] M. M. Alibegov, Phys. Rev. E 59, 4841 (1999).

[23] C. J. Tessone, H. S. Wio, and P. Hanggi, Phys. Rev. E 62, 4623 (2000).

[24] H. E. Plesser and T. Geisel, Phys. Rev. E 63, 031916 (2001).

[25] A. A. Zaikin, J. Kurths, and L. Schimansky-Geier, Phys. Rev. Lett. 85, 227 (2000).

[26] Y. Jia, S. Yu, and J. Li, Phys. Rev. E 62, 1869 (2000).

[27] Y. Jia, X. Zheng, X. Hu, and J. Li, Phys. Rev. E 63, 031107 (2001).

[28] X. M. Liu, H. Z. Xie, L. G. Liu, and Z. B. Li, Phys. A 388, 392 (2009).

[29] P. Smolen, D. A. Baxter, J. H. Byrne, Amer. J. Physiol. Cell. Physiol. 274, 531 (1998); 277 (1999) C777.

[30] C. L. Zhu, Y. Zheng, and Y. Jia, Bioph. Chem 129, 49 (2007).

[31] X. Q. Luo and S. Q. Zhu, Phys. Rev. E 67, 021104 (2003).

[32] P. Hanggi and P. Jung, Adv. Chem. Phys. 89, 239 (1995).

[33] L. Cao, D. J. Wu, and S. Z. Ke, Phys. Rev. E 52, 3228 (1995).

[34] R. F. Fox, Phys. Rev. A 34, 4525 (1986).

[35] Y. Jia and J. Li, Phys. Rev. E 53, 5786 (1996).

[36] W. R. Zhong, Y. Z. Shao, and Z. H. He, Fluc. and Noi. Lett. 6, L349 (2006).

[37] C. J. Wang and D. C. Mei, Fluc. and Noi. Lett. 8, L213 (2008).

[38] E. A. Novikov, Zh. Eksp. Teor. Fiz. 47, 1919 (1964); [Sov. Phys. JETP 29, 1290 (1965)].

[39] D. A. Day and M. F. Tuite, Jour of Endo 157, 361C371 (1998).

[40] A. Fujita, J. R. Sato, H. M. Garay-Malpartida, M. C. Sogayar, C. E. Ferreira, and S. Miyano, Jour. of Bioi. and Comp. Bio. $6,5(2008)$ 